\title{
Swimming pool attendance and risk of asthma and allergic symptoms in children
}

\author{
L. Font-Ribera*,\#, M. Kogevinas*,\#,ף,+, J-P. Zock ${ }^{*, \#, \oplus, ~ M . J . ~ N i e u w e n h u i j s e n *, \#, ~, ~}$ \\ D. Heederik ${ }^{\S}$ and C.M. Villanueva ${ }^{*, \#, \emptyset}$
}

ABSTRACT: Increased asthma risk has been associated with pool attendance in children but evidence is inconsistent and inconclusive. A survey was conducted of 3,223 9-12-yr-old children in Sabadell (Spain) to evaluate association between swimming pool attendance and prevalence of asthma and allergic conditions and symptoms.

Parents completed a questionnaire on lifetime frequency of pool attendance and symptoms in the last 12 months (wheezing, asthma medication, rhinitis and allergic rhinitis), ever having asthma and eczema, and potential confounders. Indicators of indoor and outdoor swimming pool attendance early in life, cumulatively and currently were calculated.

Swimming pool attendance before the age of 2 yrs was associated with slightly lower prevalence of current asthma (OR $0.79,95 \% \mathrm{Cl} 0.43-1.46)$, rhinitis (OR $0.86,95 \% \mathrm{Cl} 0.68-1.08$ ) and allergic rhinitis symptoms (OR $0.72,95 \% \mathrm{Cl} 0.54-0.96$ ) compared to those who started attending swimming pools after $4 \mathrm{yrs}$ of age. An increased prevalence of eczema was associated with duration of lifetime pool attendance (OR $1.71,95 \% \mathrm{Cl} 1.38-2.12$ for $>5 \mathrm{yrs}$ versus $0 \mathrm{yrs}$ ).

Swimming pool attendance in Spanish children was associated with slightly less upper and lower respiratory tract symptoms and with more eczema. Longitudinal studies are required to confirm these findings and avoid potential reverse causation.

KEYWORDS: Allergic symptoms, asthma, children, eczema, rhinitis, swimming pools

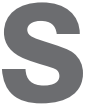
wimming is extensively practised in Spain and other western countries, where sedentarism is increasing [1, 2]. Besides the benefits of physical activity, swimming in pools involves exposure to disinfectants and disinfection by-products. The most widely used disinfectants are chlorine based. Trichloramine is a volatile and irritant chlorination by-product present in swimming pools, believed to be responsible for the characteristic odour and irritant symptoms $[3,4]$.

Acute respiratory effects, eye irritation and rash have been described in recreational swimmers after accidental exposure to high doses of chlorine $[5,6]$. Chronic exposure to swimming pools has been associated with several respiratory symptoms and irritant eye, nasal and throat symptoms among pool workers [7]. Furthermore, cases of occupational asthma have been described in pool lifeguards [8]. Respiratory symptoms and asthma among competitive swimmers are more prevalent compared to other athletes [9]. Although the World Health Organization (WHO) sets guidelines for safe recreational water [4], the potential risk of inducing or exacerbating childhood asthma among those attending even well-kept swimming pools is a concern. Recent studies attempted to elucidate whether swimming in pools is a risk factor for asthma and other atopic diseases in children [10]. Although some studies reported a positive association between chlorinated swimming pool attendance and asthma prevalence, particularly among atopic children [11, 12], other studies have not replicated these findings [13, 14]. Overall, current evidence associating childhood asthma and swimming pool attendance is inconsistent and inconclusive [10].

Atopic diseases, such as asthma and eczema, are among the most common chronic diseases in children, and prevalence is increasing [15]. Multiple factors could explain this increase, including environmental changes (new chemicals or allergens), patterns of infection early in life,

Earn CME accreditation by answering questions about this article. You will find these at the back of the printed copy of this issue or online at www.erj.ersjournals.com/misc/cmeinfo.dtl

AFFILIATIONS

${ }^{*}$ Centre for Research in

Environmental Epidemiology (CREAL),

${ }^{\#}$ Municipal Institute of Medical Research (IMIM-Hospital del Mar), and

"CIBER Epidemiología y Salud Pública (CIBERESP), Barcelona, Spain.

+Dept of Social Medicine, Medical School, University of Crete, Crete, Greece.

${ }^{\text {s}}$ Division of Environmental Epidemiology, Institute for Risk Assessment Sciences, Utrecht University, Utrecht, The Netherlands.

CORRESPONDENCE

C.M. Villanueva

Centre for Research in Environmental Epidemiology (CREAL)

Doctor Aiguader

88

08003-Barcelona

Spain

E-mail: cvillanueva@creal.cat

Received:

Nov 282008

Accepted after revision:

April 282009

First published online:

May 142009 
and lifestyle (e.g. decreased physical activity, nutrition and living environment) [16].

In order to contribute new data to this important public health issue, we conducted a study of $>3,200$ children in a southern European population, where both indoor and outdoor pool attendance are very common. We evaluated the association between indoor and outdoor swimming pool attendance during different time-periods and the prevalence of asthma and allergic symptoms and conditions in children.

\section{METHODS}

\section{Study design and population}

In June 2006 we conducted a cross-sectional population-based study in the municipality of Sabadell, Spain $(\sim 200,000$ inhabitants). We aimed to recruit all children in the 4 th, 5 th and 6th year of primary school (9-12 yr olds) in the 58 primary schools of the municipality, 53 of which agreed to participate. Questionnaires were provided to the parents of the children. The overall response rate was 58\% (3,322 questionnaires). School-specific response rates were $>50 \%$ for 36 schools, and for these schools the response rate was $67 \%$. General characteristics and the prevalence of the health outcomes under study did not differ between these 36 schools and the 17 schools with response rates $<50 \%$. Therefore, we combined data from both high- and low-response schools in the analysis. The study was approved by the ethics committee of the research centre and signed informed consent was provided by all participants.

In order to evaluate the level of the irritants in the swimming pools of the study area, we contacted the nine indoor swimming pools and two outdoor pools in Sabadell. The concentration of trichloramine was measured in air samples of the indoor pools over a $2-\mathrm{h}$ period, and again 2 weeks later over two consecutive 2 -h periods. In outdoor pools a single sample was obtained. In total, 29 samples were collected. Sampling points were located within $1 \mathrm{~m}$ from the water and at a height of $40-50 \mathrm{~cm}$ from the floor level. The method used for trichloramine sampling and analysis has been described previously by Héry et al. [17]. Briefly, air was pumped with a 1.2 L. $\mathrm{min}^{-1}$ flow through two quartz fibre filters impregnated with a diarsenic trioxide solution. After treatment, trichloramines were reduced to chlorides and subsequently analysed by ion chromatography. The experimental analyses were performed in the Institute for Risk Assessment Sciences at Utrecht University (Utrecht, the Netherlands) and further details have been presented previously [3]. Free chlorine and bromine in the water were also recorded.

\section{Questionnaire}

Information was collected through a parent-administrated questionnaire. Swimming pool attendance data was obtained using categorical questions on the frequency of current attendance to indoor and outdoor swimming pools in summer or winter and attendance during different periods since birth. Winter referred to the school term (mid-September to mid-June), and summer referred to the summer holidays period (mid-June to mid-September). The following variables for swimming pool attendance were created: swimming pool attendance (ever/ never); age when started attending pools $(<2,2-4,>4$ yrs); years attending pools in summer $(\leqslant 5,6-9,>9$ yrs $)$; years attending pools in winter $(0,1-5,>5$ yrs); current pool attendance in the last 12 months (never, at least once a month in summer, at least once a month in summer and in winter). We also asked for the name of the swimming pools that the children had attended during the last 12 months.

The respiratory health status of children was assessed using questions from the validated International Study of Asthma and Allergies in Childhood (ISAAC) questionnaire, translated into Spanish and Catalan [18]. We asked about "asthma ever", followed by the questions "has it been diagnosed by a physician?" and "at what age was he or she diagnosed with asthma?"; we also asked about "asthma medication in the last 12 months" and "wheezing in the last 12 months". "Current asthma" was defined as asthma ever plus "having had wheezing or having used asthma medication in the last 12 months". "Current rhinitis symptoms" (sneezing, runny or blocked nose without cold in the last 12 months) and "current allergic rhinitis symptoms" (sneezing, runny or blocked nose without cold in the last 12 months plus itchywatery eyes in the last 12 months) were also considered as outcome variables. We asked about "eczema or atopic dermatitis ever", as a proxy for atopic status, to evaluate potential effect modification. Finally, "eczema ever" was also evaluated as an outcome variable.

Two questions were included to evaluate whether swimming pool attendance was influenced by respiratory or allergic health. The question: "If your child is currently not attending a pool, what is the main reason? 1) practices other sports, 2) health problems (respiratory, allergies, skin irritation, others), 3) other reasons"; was aimed to detect pool avoidance due to respiratory, allergic or skin problems (negative selection). The question: "Is the reason why your child currently goes to the pool related to a health condition? 1) no, 2) yes, respiratory or allergic condition, 3) yes, backache, obesity or other"; was aimed to detect children that attended pools because of respiratory problems (positive selection). 102 children whose swimming pool attendance was related to respiratory or allergic conditions were excluded from the multivariate analyses. Other information was collected on sex, age, weight and height, daily hours spent in front of the TV (a proxy for sedentarism), current sports, parental asthma and educational level. In order to evaluate the questionnaire reproducibility, we telephoned a subset of 60 families who were re-contacted 2 months later asking for swimming pool attendance and symptoms. The agreement rates between the first and the second interviews were $85 \%$ for pool attendance in the past, $92 \%$ for current attendance and $92 \%$ for symptoms.

\section{Statistical analysis}

A descriptive analysis and a multivariate logistic regression analysis were performed with the statistical package STATA 8.2 to estimate adjusted odds ratios (ORs) and 95\% confidence intervals (CIs) of the respiratory outcomes associated with different indicators of swimming pool attendance. We selected potential confounders on the basis of previous evidence and retained these factors in the models if they substantially $(>5 \%)$ modified ORs. The statistical analyses excluded 39 children with missing data on age or sex. As eczema status appeared to be a relevant variable included in all the final models (either as an outcome or confounder), 60 children with missing eczema 


TABLE $1 \quad$ Characteristics of the study population,
prevalence of health outcomes and swimming
pool attendance

Data are presented as $n(\%)$, unless otherwise stated. Numbers do not always add to a total of 3,223 because of missing information. ${ }^{\#}$ : defined as asthma ever with medication or wheezing in the last 12 months; ': refers to midSeptember until mid-June (i.e. mainly indoor pools); ${ }^{+}$: refers to mid-June until mid-September (i.e. mainly outdoor pools).

status were also excluded, and the final study population was 3,223 . Among those, asthma status, current rhinitis and current allergic rhinitis symptoms were missing in 32, 20 and 22 children, respectively.

\section{RESULTS}

The characteristics of the study population and the prevalence of symptoms are shown in table 1 . Among the $7.1 \%$ of children who reported having ever asthma, 92\% were physician diagnosed. The mean $\pm \mathrm{SD}$ age for asthma diagnosis was $3.7 \pm 2.9$ yrs. The prevalence of asthma, rhinitis symptoms and eczema varied with sex, body mass index (BMI) and watching TV (results not shown). Asthma prevalence was higher in younger males and increased with BMI. Rhinitis symptoms increased with BMI and with time spent watching TV. Parental asthma was positively associated with all the outcomes, whereas low parental education was associated with a higher prevalence of current respiratory and rhinitis symptoms, but with a lower prevalence of eczema.

Only $81(2 \%)$ children reported never attending swimming pools. Lifetime winter attendance was mainly indoors in $93 \%$ of children, and equally indoors and outdoors in $4 \%$. Summer attendance in children was mainly outdoors in $85 \%$ and mainly indoors in $3 \%$. Years of pool attendance in winter correlated with years of summer attendance $(p<0.001$, value for the Spearman correlation). Swimming pool attendance was positively associated with parental education, but did not differ by sex, age or parental asthma. Children who started attending pools before age 4 yrs had lower current BMI and spent less hours watching TV (table 2).

Seven indoor swimming pools used chlorine as disinfectant, and bromine was used in two swimming pools. Most of the children (95\%) attended only chlorinated swimming pools in the last 12 months, $2 \%$ attended only brominated pools and $3 \%$ attended both. Trichloramine levels are shown in figure 1 . The median level was $0.160 \mathrm{mg} \cdot \mathrm{m}^{-3}$ in the seven chlorinated pools, $0.026 \mathrm{mg} \cdot \mathrm{m}^{-3}$ in the two brominated pools and 100 -fold lower in the two outdoor chlorinated pools. Free chlorine in water ranged from 0.20 to $2.80 \mathrm{mg} \cdot \mathrm{L}^{-1}$ in the chlorinated pools (median level $1.25 \mathrm{mg} \cdot \mathrm{L}^{-1}$ ).

The association between swimming pool attendance and asthma is shown in table 3. Crude and adjusted ORs were similar and we only present estimates adjusted for eczema and/or parental educational level. Further adjustment for BMI or watching TV did not change the ORs. Swimming pool attendance was slightly related to a lower prevalence of current asthma. The OR of current asthma for those currently attending pools was 0.46 (95\% CI $0.25-0.83)$. A similar pattern was observed for ever asthma, but these inverse associations did not reach statistical significance. Analyses evaluating doctor-diagnosed asthma showed similar results. The most highly exposed children (i.e. those attending swimming pools in winter for $>4$ days a week during a period in their life) did not show an increased asthma risk (OR 0.96, 95\% CI 0.51-1.78). Prevalence of current wheezing and current asthma medication also decreased with pool attendance but did not reach statistical significance (data not shown). Swimming pool attendance seemed to be a protective factor for current rhinitis and allergic rhinitis symptoms (table 4). The number of years attending swimming pools in winter, and even more clearly in summer, was associated with less rhinitis and allergic rhinitis symptoms in the last 12 months.

Stratification by sex or parental asthma did not modify the results. Stratification by type of swimming pool was not possible as brominated pool attendance was very rare. Eczema status did not modify the association between pool attendance 
TABLE 2 Determinants of swimming pool attendance in different time-periods

Total population

Early Swimming

$<4$ yrs

Sex

Female $1710(53.0)$

Male

p-value

Age yrs

8-9

10

11

12-15

$\mathrm{p}$-value

Parental education

Primary

Secondary

University

p-value

Parental asthma

No

Yes

$\mathrm{p}$-value

BMI $\mathbf{k g} \cdot \mathbf{m}^{-2}$

$<16.5$

16.5-20.5

$>20.5$

$\mathrm{p}$-value

Time watching TV $\mathbf{h} \cdot$ day $^{-1}$

$<4$

$>4$

$\mathrm{p}$-value

Total
$1513(46.9)$

$670(20.8)$

1045 (32.4)

989(30.7)

$519(16.1)$

$641(20.4)$

1439 (45.8)

1062 (33.8)

2784 (88.2)

$373(11.8)$

728 (25.9)

$1336(47.5)$

$750(26.6)$

1910 (59.5)

1299 (40.5)

3223
867 (55.5)

$798(57.2)$

0.33

369 (60.1)

535 (56.0)

510 (56.2)

251 (52.2)

0.07

$275(47.7)$

$763(56.5)$

599 (62.4)

$<0.001$

1446 (56.6)

194 (56.2)

0.90

401 (60.2)

$690(56.0)$

$373(53.1)$

0.03

$1025(58.3)$

637 (53.5)

0.01

1665 (56.3)
Attend pools in winter

for $>5$ yrs

Current attendance in

Data are presented as $n(\%)$, unless otherwise stated. Numbers do not always add to a total of 3,223 because of missing information. BMI: body mass index.

and respiratory outcomes. Among children with eczema, the OR of asthma ever in those attending pools in winter for $>5$ yrs compared to nonattendants in winter was $0.62(95 \% \mathrm{CI}$ 0.33-1.15). Asthma ever did not modify the negative association between pool attendance and current respiratory outcomes. Asthmatic children who attended pools in winter for $1-$ 5 yrs had less current wheezing (OR 0.51, 95\% CI 0.23-1.13), rhinitis symptoms (OR $0.35,95 \%$ CI $0.15-0.81$ ) and allergic rhinitis symptoms (OR $0.35,95 \%$ CI $0.15-0.81$ ) than asthmatic children who never attended pools in winter. However, these negative associations disappeared for children who had attended swimming pools for $>5$ yrs in winter. Current pool attendance was also associated with less current wheezing in asthmatic children (OR $0.22,95 \%$ CI $0.09-0.53$ for attendance only in summertime and OR $0.31,95 \%$ CI $0.11-0.86$ for attendance in summer and winter).

Swimming pool attendance consistently increased the risk of eczema ever (table 4), both for years of attendance in winter (mainly indoors) and summer (mainly outdoors), also after stratification by parental education. In addition, after adjusting for swimming pool attendance during winter, the association between duration of summer attendance and eczema ever remained (OR 1.37, 95\% CI 1.07-1.74 and OR 1.51, 95\% CI 1.201.91 for 6-9 yrs and for $>9$ yrs, respectively, compared with 0 5 yrs).

\section{DISCUSSION}

Our results indicate that swimming pool attendance was associated with a slightly lower prevalence of asthma, current rhinitis and allergic rhinitis symptoms. However, attendance at both indoor and outdoor swimming pools was associated with an increased prevalence of eczema in children.

We used different pool exposure indicators including early, cumulative and current pool attendance in summer and winter (a proxy for indoor and outdoor pools respectively). Although correlated, these indicators represent different time windows with diverse vulnerability and exposure levels [12]. Never-pool attendants were a small and possibly selected group, but this indicator may be useful for comparisons with previous studies [14]. Exposure misclassification that may have occurred is likely to be nondifferential. Recall bias is not a main concern as parents were not informed about the evaluated hypothesis and were probably unaware of it. Consequently, differential reporting of swimming pool attendance by health status was unlikely. 


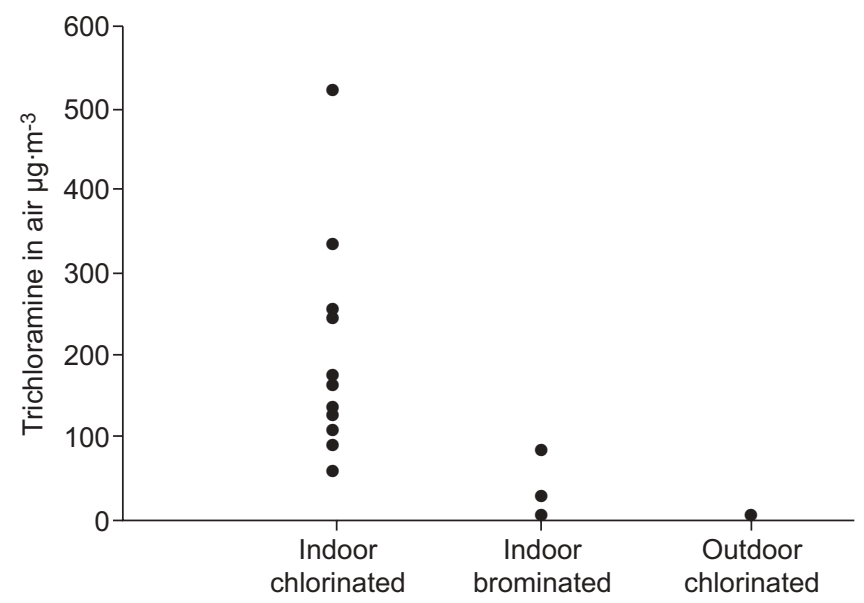

FIGURE 1. Trichloramine concentration in the air of the swimming pools in the municipality. Trichloramine in air was measured three times in the seven indoor chlorinated swimming pools ( $n=21$ measurements), three times in the two brominated swimming pools ( $n=6$ measurements) and once in two outdoor chlorinated swimming pools ( $n=2$ measurements). Median level of trichloramine in air was $160 \mu \mathrm{g} \cdot \mathrm{m}^{-3}$ in chlorinated indoor pools, $26 \mu \mathrm{g} \cdot \mathrm{m}^{-3}$ in brominated indoor pools and $2 \mu \mathrm{g} \cdot \mathrm{m}^{-3}$ in chlorinated outdoor pools.

In addition, questions on swimming pool attendance were highly reproducible. Some degree of outcome misclassification may be present as we did not have clinical measurements of respiratory and allergic symptoms. Prevalence of asthma medication was higher than asthma ever prevalence, as this treatment can be prescribed for acute respiratory conditions other than asthma (e.g. bronchitis). We had a fair response rate probably because the survey was conducted in June, close to the end of the academic year, which can be a busy period for the schools. However, the prevalence of symptoms in this population-based study was comparable with prevalence rates in equivalent Spanish populations suggesting that selection bias was unlikely.

The possibility of reverse causation is a main concern, as asthma or eczema status could influence swimming pool attendance in both directions. Children with eczema or asthma may avoid pools if symptoms are exacerbated by swimming. However, medical praxis encourage patients with eczema or asthma to be as normally active as possible [19] and swimming is a well tolerated sport among these patients [20]. Our data show that the proportion of current swimming or practice of other sports is higher among children who ever had eczema compared with other children. In order to reduce the potential selection bias, we excluded 102 children reporting attendance or avoidance of pools related to health conditions from the analyses, and the results remained practically unchanged. However, given the retrospective nature of the data collected, underreporting of swimming pool avoidance or attendance related to health outcomes in the past may have occurred and reverse causation cannot be totally ruled out.

Pool attendance tended to be a protective factor for current asthma, current rhinitis and allergic rhinitis symptoms. Interestingly, these associations were similar for children with and without eczema, which we considered a proxy for atopic

\begin{tabular}{|c|c|c|}
\hline \multirow[t]{2}{*}{ TABLE 3} & \multicolumn{2}{|c|}{$\begin{array}{l}\text { Asthma associated with different indicators of } \\
\text { swimming pool attendance }\end{array}$} \\
\hline & Asthma ever & Current asthma \\
\hline \multicolumn{3}{|c|}{ Swimming pool attendance } \\
\hline Never & 1 & 1 \\
\hline Ever & $0.53(0.25-1.12)$ & $0.29(0.13-0.61)$ \\
\hline Subjects n & 3087 & 3087 \\
\hline \multicolumn{3}{|c|}{$\begin{array}{l}\text { Age when started attending } \\
\text { pools yrs }\end{array}$} \\
\hline$<2$ & $1.09(0.71-1.67)$ & $0.79(0.43-1.46)$ \\
\hline $2-4$ & $0.90(0.64-1.28)$ & $0.92(0.59-1.43)$ \\
\hline$>4$ & 1 & 1 \\
\hline Subjects $n^{\circ}$ & 2836 & 2836 \\
\hline \multicolumn{3}{|c|}{ Years attending pools in winter ${ }^{+}$} \\
\hline 0 & 1 & 1 \\
\hline $1-5$ & $0.92(0.62-1.37)$ & $0.66(0.39-1.09)$ \\
\hline$>5$ & $0.82(0.55-1.21)$ & $0.77(0.47-1.26)$ \\
\hline Subjects $n^{\bullet}$ & 2898 & 2898 \\
\hline \multicolumn{3}{|c|}{$\begin{array}{l}\text { Years attending pools in } \\
\text { summer }^{\S}\end{array}$} \\
\hline $0-5$ & 1 & 1 \\
\hline $6-9$ & $0.90(0.60-1.35)$ & $0.79(0.47-1.33)$ \\
\hline$>9$ & $0.83(0.57-1.21)$ & $0.79(0.49-1.27)$ \\
\hline Subjects $n^{\bullet}$ & 2716 & 2716 \\
\hline \multicolumn{3}{|c|}{$\begin{array}{l}\text { Current attendance in the last } \\
12 \text { months }\end{array}$} \\
\hline Never & 1 & 1 \\
\hline Only in summer & $0.78(0.52-1.18)$ & $0.44(0.27-0.69)$ \\
\hline Summer and winter & $0.68(0.41-1.14)$ & $0.46(0.25-0.83)$ \\
\hline Subjects $n^{*}$ & 3020 & 3020 \\
\hline
\end{tabular}

Data are presented as odds ratio ( $95 \%$ confidence interval), unless otherwise stated. Odds ratios are adjusted for eczema ever. ${ }^{\#}$ : defined as asthma ever with medication or wheezing in the last 12 months; ' number of children included in the model varies because of missing data on the outcome or exposure variables. 102 children who reported attendance related to health conditions were excluded from the model; ${ }^{+}$: refers to mid-September until midJune (i.e. mainly indoor pools); ${ }^{\text {s: }}$ refers to mid-June until mid-September (i.e. mainly outdoor pools).

status. The increased risk of asthma described in studies carried out in children in Belgium was statistically significant mainly in atopic children defined by serum specific immunoglobin (Ig)E levels [11]. In our southern European population, outdoor pool attendance is very common, involving much lower exposure to volatiles such as trichloramine. When considering only wintertime pool attendance, a proxy for indoor pools, we still did not find an increased asthma risk. Our results are consistent with studies conducted in other countries [13, 14], including a German cohort where a protective effect on asthma prevalence at 6 yrs was found for early pool attendance [13]. Furthermore, a very recent metaanalysis on pool attendance and asthma prevalence in children found an OR of 0.82 (95\% CI 0.54-1.25), decreasing to OR 0.63 (95\% CI 0.38-1.06) when only the studies with higher quality were considered [9]. We speculate that physical activity during swimming could improve respiratory health and partly explain this protective effect. Recent literature shows that 

TABLE 4 Rhinitis symptoms in last 12 months and eczema ever associated with different indicators of swimming pool
attendance

Current rhinitis symptoms

Current allergic rhinitis symptoms

$0.69(0.44-1.09)$

3099

$0.86(0.68-1.08)$

$0.85(0.71-1.01)$

1

2843

Subjects $\mathrm{n}^{\#}$

Years attending pools in winter

0

$1-5$

$>5$

Subjects $\mathrm{n}^{\#}$

Years attending pools in summer ${ }^{+}$

$0-5$

6-9

$>9$

Subjects $\mathrm{n}^{\#}$

Current attendance in the last 12 months

Never

Only in summer

Summer and winter

Subjects $\mathrm{n}^{\#}$
1

$0.84(0.68-1.03)$

$0.92(0.75-1.14)$

2915

$0.79(0.64-0.98)$

$0.84(0.69-1.02)$

2723

1

$0.91(0.73-1.14)$

0.90 (0.69-1.17)

3032
$0.56(0.34-0.94)$

3097

$0.72(0.54-0.96)$

$0.76(0.61-0.94)$

1

2842

1

$0.79(0.61-1.01)$

$0.82(0.64-1.06)$

2908

1

$0.75(0.58-0.98)$

$0.70(0.55-0.90)$

2718

1

$0.76(0.55-1.04)$

3029
$0.74(0.57-0.97)$
Eczema ever

1

$1.84(1.05-3.21)$

3058

$1.00(0.80-1.26)$

$1.01(0.85-1.20)$

1

2862

1

$1.70(1.37-2.11)$

$1.71(1.38-2.12)$

2927

1

$1.43(1.16-1.78)$

$1.50(1.23-1.83)$

2738

1

$1.38(1.09-1.74)$

$1.69(1.29-2.21)$

3051

Data are presented as adjusted odds ratio (95\% confidence interval), unless otherwise stated. Current rhinitis and allergic rhinitis symptom models adjusted for eczema ever and parental educational level. Eczema ever models adjusted for parental educational level. ${ }^{*}$ : number of children included in the model varies because of missing data on the outcome or exposure variables. 102 children who reported attendance related to health conditions were excluded from the model;" : refers to mid-September until mid-June (i.e. mainly indoor pools); ${ }^{+}$: refers to mid-June until mid-September (i.e. mainly outdoor pools).

physical activity during childhood [21, 22] or adulthood [23] could reduce hay fever [21], bronchial hyperresponsiveness [23] and asthma development [22]. Future studies should make an effort to properly measure physical activity as a main potential confounder.

The levels of trichloramine in indoor pool air in our study population (median $0.16 \mathrm{mg} \cdot \mathrm{m}^{-3}$ ) were lower than the WHO recommendations $\left(<0.5 \mathrm{mg} \cdot \mathrm{m}^{-3}[4]\right)$. Levels were also lower compared to published levels from Belgium $\left(0.325 \mathrm{mg} \cdot \mathrm{m}^{-3}\right)$ [12], France $\left(0.240-0.670 \mathrm{mg} \cdot \mathrm{m}^{-3}\right)$ [7] or the Netherlands $\left(0.565 \mathrm{mg} \cdot \mathrm{m}^{-3}\right)$ [3]. However, annual average levels could have been underestimated as measurements were conducted in summertime, when ventilation is higher and the number of swimmers in indoor pools is lower. The legislation in the study area sets the level of free chlorine in water of swimming pools in the range of $0.5-2 \mathrm{ppm}$ [24]. As the eventual asthma risk is probably dose-dependent, the different levels of irritants in the swimming pool environment could explain the inconsistent results among studies.

We found a statistically significant increased risk of eczema associated with indoor and outdoor cumulative pool attendance, which has not been previously reported. Eczema (or atopic dermatitis) is a chronic inflammatory skin disease characterised by the formation of specific antibodies (IgE) to environmental allergens, which frequently starts early in infancy and affects $15-30 \%$ of children in industrialised countries [25]. Although IgE blood levels were unrelated to both cumulative and acute pool exposure in two studies [26, 27], a semi-experimental study suggested that dermal pool exposure may play a role in the exacerbation of atopic dermatitis [28]. Furthermore, it has been described that workers engaged in wet-works are at risk for suffering irritant dermatitis [29], which in turn may enhance the atopic dermatitis [30]. It should be noted that in the two studies where pool attendance was positively associated with increased asthma risk [11, 12], the asthma definition was partly based on the level of fractional exhaled nitric oxide $(F$ eNO), a marker of allergic airway inflammation. However, FeNO is not specific for asthma and it may be an indicator of atopic diseases including atopic dermatitis or eczema [31].

In conclusion, swimming pool attendance in Spanish children was slightly associated with less upper and lower respiratory tract symptoms and with more eczema. Our results do not support the hypothesis that swimming pool attendance increases the risk of asthma among children. However, longitudinal studies are required to confirm these findings and to avoid potential reverse causation. 


\section{SUPPORT STATEMENT}

This work has been funded by the Spanish Ministry of Education and Science (Plan National Grant SAF2005-07643-C03-01). L. Font-Ribera has a predoctoral fellowship from the Spanish Health Ministry (FI06/ 00651). C.M. Villanueva and J-P. Zock have a contract funded by the Instituto de Salud Carlos III, Spanish Ministry of Health and Consumption (CP06/00341 and 01/3058, respectively)

\section{STATEMENT OF INTEREST}

None declared.

\section{ACKNOWLEDGEMENTS}

We thank T. Corbella (Municipal Council, Sabadell, Spain), L. Arjona (CREAL, Barcelona, Spain) for her contribution in the fieldwork, A. Novoa (CREAL) for her contribution to the fieldwork and data analysis in some parts of the study, K. Meliefste (Division of Environmental Epidemiology, Institute for Risk Assessment Sciences, Utrecht University, Utrecht, the Netherlands) for carrying out the trichloramine analyses and D. MacFarlane (IMIM-Hospital del Mar, Barcelona) for the English revision.

\section{REFERENCES}

1 Vaz de Almeida MD, Graca P, Afonso C, et al. Physical activity levels and body weight in a nationally representative sample in the European Union. Public Health Nutr 1999; 2: 105-113.

2 Kafatos A, Manios Y, Markatji I, et al. Regional, demographic and national influences on attitudes and beliefs with regard to physical activity, body weight and health in a nationally representative sample in the European Union. Public Health Nutr 1999; 2: 87-95.

3 Jacobs JH, Spaan S, van Rooy GB, et al. Exposure to trichloramine and respiratory symptoms in indoor swimming pool workers. Eur Respir J 2007; 29: 690-698.

4 World Health Organization. Guidelines for Safe Recreational Water Environments. Volume 2. Swimming Pools and Similar Environments. World Health Organization, Geneva, 2006. www. who.int/water_saniation_health/bathing/srwe2full.pdf.

5 Bonetto G, Corradi M, Carraro S, et al. Longitudinal monitoring of lung injury in children after acute chlorine exposure in a swimming pool. Am J Respir Crit Care Med 2006; 174: 545-549.

6 Kaydos-Daniels SC, Beach MJ, Schwe T, et al. Health effects associated with indoor swimming pools: a suspected toxic chloramine exposure. Public Health 2008; 122: 195-200.

7 Massin N, Bohadana AB, Wild P, et al. Respiratory symptoms and bronchial responsiveness in lifeguards exposed to nitrogen trichloride in indoor swimming pools. Occup Environ Med 1998; 55: 258-263.

8 Thickett KM, McCoach JS, Gerber JM, et al. Occupational asthma caused by chloramines in indoor swimming-pool air. Eur Respir J 2002; 19: 827-832.

9 Goodman M, Hays S. Asthma and swimming: a meta-analysis. J Asthma 2008; 45: 639-647.

10 Weisel CP, Richarson SD, Nemery B, et al. Childhood asthma and environmental exposures at swimming pools: state of the science and research recommendations. Environ Health Prespec 2009; 117: 500-507.

11 Bernard A, Carbonnelle S, De Burbure C, et al. Chlorinated pool attendance, atopy, and the risk of asthma during childhood. Environ Health Perspect 2006; 114: 1567-1573.

12 Bernard A, Carbonnelle S, Dumont X, et al. Infant swimming practice, pulmonary epithelium integrity, and the risk of allergic and respiratory diseases later in childhood. Pediatrics 2007; 119: 1095-1103.

13 Schoefer Y, Zutavern A, Brockow I, et al. Health risks of early swimming pool attendance. Int J Hyg Environ Health 2007; 211: 367-373.

14 Kohlhammer $\mathrm{Y}$, Doring $\mathrm{A}$, Schafer $\mathrm{T}$, et al. Swimming pool attendance and hay fever rates later in life. Allergy 2006; 61: 1305-1309.

15 O'Connell EJ. The burden of atopy and asthma in children. Allergy 2004; 59: Suppl. 78, 7-11.

16 Lucas SR, Platts-Mills TA. Physical activity and exercise in asthma: relevance to etiology and treatment. J Allergy Clin Immunol 2005; 115: 928-934.

17 Hery M, Hecht G, Gerber JM, et al. Exposure to chloramines in the atmosphere of indoor swimming pools. Ann Occup Hyg 2008; 39: 427-439.

18 Mata Fernández C, Fernández-Benitez M, Pérez Miranda M, et al. Validation of the Spanish version of the Phase III ISAAC questionnaire on asthma. J Investig Allergol Clin Immunol 2005; 15: 201-210.

19 American College of Sports MedicineACSM's Guidelines For Exercise Testing And Prescription. 6th Edn. Philadelphia, Lippencott Williams \& Wilkins, 2000.

20 Wardell CP, Isbister C. A swimming program for children with asthma. Does it improve their quality of life? Med J Aust 2000; 173: 647-648.

21 Kohlhammer $Y$, Zutavern A, Rzehak $P$, et al. Influence of physical inactivity on the prevalence of hay fever. Allergy 2006; 61: 1310-1315.

22 Rasmussen F, Lambrechtsen J, Siersted HC, et al. Low physical fitness in childhood is associated with the development of asthma in young adulthood: the Odense schoolchild study. Eur Respir J 2000; 16: 866-870.

23 Shaaban R, Leynaert B, Soussan D, et al. Physical activity and bronchial hyperresponsiveness: European Community Respiratory Health Survey II. Thorax 2007; 62: 403-410.

24 Generalitat de Catalunya. Decret 95/2000, de 22 de febrer, pel qual s'estableixen les normes sanitàries aplicables a les piscines d'ús public [Decree 29/2000 for health regulation in public swimming pools]. www.gencat.cat/salut/depsalut/pdf/decret95.pdf Date last accessed: October 1, 2009. Date last updated: February 22, 2000.

25 Bieber T. Atopic dermatitis. N Engl J Med 2008; 358: 1483-1494.

26 Bernard A, Carbonnelle S, Michel O, et al. Lung hyperpermeability and asthma prevalence in schoolchildren: unexpected associations with the attendance at indoor chlorinated swimming pools. Occup Environ Med 2003; 60: 385-394.

27 Carbonnelle S, Francaux M, Doyle I, et al. Changes in serum pneumoproteins caused by short-term exposures to nitrogen trichloride in indoor chlorinated swimming pools. Biomarkers 2002; 7: 464-478.

28 Seki T, Morimatsu S, Nagahori H, et al. Free residual chlorine in bathing water reduces the water-holding capacity of the stratum corneum in atopic skin. J Dermatol 2003; 30: 196-202.

29 English JS. Current concepts of irritant contact dermatitis. Occup Environ Med 2004; 61: 722-726.

30 English J. Current concepts in contact dermatitis. Br J Dermatol 2001; 145: 527-529.

31 Welsh L, Lercher P, Horak E. Exhaled nitric oxide: interactions between asthma, hayfever, and atopic dermatitis in school children. Pediatr Pulmonol 2007; 42: 693-698. 